\title{
BMJ Open Mapping of modifiable barriers and facilitators of medication adherence in bipolar disorder to the Theoretical Domains Framework: a systematic review protocol
}

\author{
Asta Ratna Prajapati, ${ }^{\oplus 1,2}$ Alexandra Lelia Dima, ${ }^{\circ}$ Allan B Clark, ${ }^{4}$ Claire Gant, ${ }^{5}$ \\ Chris Gibbons, ${ }^{6}$ Richard Gorrod, ${ }^{5}$ George Mosa, ${ }^{7}$ Sion Scott, ${ }^{2}$ Fujian Song, ${ }^{4}$ \\ Bonnie Teague, ${ }^{7}$ Michael J Twigg, ${ }^{2}$ Jon Wilson, ${ }^{7}$ Debi Bhattacharya ${ }^{2}$
}

To cite: Prajapati AR, Dima AL, Clark AB, et al. Mapping of modifiable barriers and facilitators of medication adherence in bipolar disorder to the Theoretical Domains Framework: a systematic review protocol. BMJ Open 2019;9:e026980. doi:10.1136/ bmjopen-2018-026980

- Prepublication history for this paper is available online. To view these files, please visit the journal online (http://dx.doi. org/10.1136/bmjopen-2018026980).

Received 29 September 2018

Revised 2 January 2019

Accepted 8 January 2019
Check for updates

(C) Author(s) (or their employer(s)) 2019. Re-use permitted under CC BY-NC. No commercial re-use. See rights and permissions. Published by BMJ.

For numbered affiliations see end of article.

Correspondence to

Asta Ratna Prajapati;

asta.prajapati@nsft.nhs.uk

\section{ABSTRACT}

Introduction People with bipolar disorder require long-term treatment but it is estimated that $40 \%$ of these people do not adhere to prescribed medication regimens. Non-adherence increases the risk of relapse, hospitalisation and suicide. Some evidence syntheses report barriers to mental health treatment adherence but rarely delineate between modifiable and non-modifiable barriers. They also fail to distinguish between the patients' perspective and that of other stakeholders such as clinicians despite of their different understanding and priorities about adherence. Facilitators of adherence, which are also important for informing adherence intervention design, are also lacking from syntheses and few syntheses focus on medications for bipolar disorder. This systematic review aims to identify modifiable barriers and facilitators (determinants) of medication adherence in bipolar disorder. We also plan to report determinants of medication adherence from perspectives of patients, carers, healthcare professionals and other third parties. A unique feature of this systematic review in the context of mental health is the use of the Theoretical Domains Framework (TDF) to organise the literature identified determinants of medication adherence.

Methods and analysis The protocol adheres to Preferred Reporting Items for Systematic Reviews and Meta-Analyses Protocols and ENhancing Transparency in REporting the synthesis of Qualitative research (ENTREQ) guidelines. This review will include both qualitative and quantitative primary studies exploring determinants of medication adherence in bipolar disorder. We will search the following databases using a preplanned strategy: CINAHL, Cochrane Library (CENTRAL), Embase, LiLACS, Medline, PsychINF0, PubMed without date restrictions. We will report the quality of included studies. We will use framework synthesis using the TDF as an a priori 'framework'. We will map the literature identified modifiable determinants to the domains of TDF. Ethics and dissemination Ethical approval is not required as primary data will not be collected. The results will be disseminated through a peer-reviewed publication. PROSPERO registration number CRD42018096306.

\section{Strengths and limitations of this study}

- As the Theoretical Domains Framework (TDF) has been mapped to evidence-based behaviour change techniques, mapping modifiable determinants of medication adherence in bipolar disorder to the TDF offer significant utility for intervention development.

- This study will provide literature identified barriers and facilitators (determinants) of medication adherence in bipolar disorder from the perspectives of patients, carers, healthcare professionals and other third parties such as researchers.

- Lack of data and quality of reporting may limit our ability to present determinants of adherence from perspectives of patients, carers, healthcare professionals and other third parties.

- Variation in the terms that is used to describe determinants of adherence may introduce a risk of mapping errors through misinterpretation of the reported barrier or facilitator.

\section{INTRODUCTION}

The lifetime prevalence of bipolar disorder is estimated at $1.4 \%$ of the UK adult population. ${ }^{1}$ Bipolar disorder, featuring mood and activity level disturbance, is a recurrent disorder and usually requires long-term maintenance therapy. ${ }^{12}$ However, it is estimated that $40 \%$ of people with bipolar disorder do not take their medication as prescribed. ${ }^{3}$ This non-adherence (generally described as taking less than $80 \%$ of prescribed doses of medication $)^{4}$ increases the risk of relapse, suicide and hospitalisation. ${ }^{56}$ For example, the probability of hospitalisation of non-adherent patients with bipolar disorder is at least five times higher than that of adherent patients. $^{7}$ 
Adhering to prescribed medication regimes is a complex health behaviour, which requires the patient to obtain the prescribed medication, have the physical and cognitive ability (practical function), and motivation (perceptual function) to take the medication. ${ }^{8}$ Furthermore, non-adherence may occur at initiation (ie, patient may or may not start the treatment), implementation (ie, patient may delay, omit or take extra doses during treatment) or persistence (ie, patient may discontinue treatment after some time) phase. ${ }^{9}$ There are many reported barriers and facilitators (determinants) of medication adherence. For this review, a barrier is defined as 'a circumstance that prevents the patients from taking their medication as prescribed', whereas a facilitator is 'a circumstance that makes the process easy or easier'. ${ }^{10}$ We mention these barriers and facilitators as 'determinants'.

The challenges to successfully address non-adherence are to:

1. Accurately identify non-adherent behaviour.

2. Determine individuals' determinants of medication adherence.

3. Select the most appropriate individualised adherence intervention(s) underpinned by health psychology theory and empirical evidence. ${ }^{11} 12$

There are various objective (eg, drug plasma levels, pill counts and electronic monitoring of medication adherence such as medication event monitoring systems) and subjective (eg, self-reported, carer or relative reported, clinician reported adherence rating scales) approaches to identify patients not adhering to their prescribed medication for bipolar disorder. ${ }^{13}$ However, there are no validated tools for comprehensively eliciting from patients and/or their carers their individual determinants of adherence to their prescribed medication for bipolar disorder. There is also an absence of theory and evidence informed guidance for practitioners to work with patients in selecting the most effective interventions for identified determinants of an individual's non-adherent behaviour.

In order to generate such a tool, there is, therefore, a need to synthesise the available evidence regarding determinants of medication adherence in patients with bipolar disorder.

A recent systematic review (literature search restricted to 1990-2015) of adherence to antipsychotic medication in bipolar disorder and schizophrenia has provided a good overview of the likely barriers experienced by people with bipolar disorder. ${ }^{14}$ However, it failed to explore factors that might facilitate adherence and excluded studies involving medication other than antipsychotics, and therefore did not identify determinants of adherence to lithium and other mood stabilisers. This is a significant omission as lithium is considered the gold-standard first-line treatment for bipolar disorder. ${ }^{15}{ }^{15}$ The determinants of adherence may be different among patients taking lithium relative to other antipsychotics due to a variety of factors including regular blood test requirements of lithium, dietary restrictions and significant interactions with other medications. Thus, a systematic review without the date limits of the previous systematic review $^{14}$ is warranted to better represent the mood stabilisers that were the mainstay of treatment in the earlier decades and not included in the previous review and to identify emerging research. ${ }^{17}$

The dearth of adherence evidence syntheses in the mental health setting underpinned by health psychology theory $^{14}{ }^{18-20}$ is of concern given its importance for informing intervention design and implementation. ${ }^{11} 12$ The Theoretical Domains Framework (TDF) is a comprehensive framework capturing 33 theories and 84 theoretical constructs related to behaviour change. ${ }^{21}$ The TDF comprises 14 domains, each of which has been coupled with evidence-based behaviour change techniques. ${ }^{22}$ The TDF therefore offers an appropriate theory for underpinning an evidence synthesis of determinants of adherence as it will enable determinants to be linked to evidencebased behaviour change techniques. This in turn will inform the development of an adherence intervention to support practitioners and patients to work together in identifying an individual's key determinants of adherence and select the most appropriate evidence-based interventions.

The perspective of patients, carers and healthcare professionals often differ in terms of the determinants of medication adherence due to differing priorities and knowledge of the situation. ${ }^{1823-26}$ For example, the healthcare professional is generally the expert regarding how the medication should be taken while the patient and carer are the experts in the patient's lived experience of taking or trying to take the prescribed medication. Furthermore, some determinants such as sex, age and ethnicity are not modifiable, and therefore have no related specific evidence-based behaviour change techniques.

A literature review matching adherence interventions to determinants of adherence concluded that adherence interventions are often not congruent with the modifiable determinants of adherence. ${ }^{27}$ We will explore the modifiable determinants of medication adherence among patients with bipolar disorder from the perspectives of the patient, carer, healthcare professional and other third parties such as researchers. For the purpose of this systematic review, we define modifiable as 'any determinants (barriers or facilitators) of medication adherence that can be modified by the patient, carer or the prescriber to improve adherence. Modifiable in the context of an individual being able to effect the change themselves or in partnership with their carer or healthcare team within a short timeframe'.

For example, knowledge about the condition/treatmentcan be changed within days or weeks. In contrast, while substance abuse can be changed over an extended period, a change is unlikely to be achievable within the timeframes acceptable for improving adherence.

This systematic review is a part of the Collaborative Medication Adherence in Bipolar disorder (C-MAB) 
project funded by Health Education England/National Institute of Health Research UK. The C-MAB project aims to develop a medication adherence tool for people with bipolar disorder. The tool is intended to identify both non-adherent behaviour and the individual's determinants of non-adherence. Following the systematic review, we will develop the tool in the form of statements derived from the literature identified modifiable determinants of adherence. We will then refine the statements by conducting focus groups and interviews with patients with bipolar disorder and their carers to better understand and prioritise the literature identified modifiable determinants. After appropriate refinement, the tool will be tested with patients with bipolar disorder.

\section{Aim}

To identify modifiable determinants of medication adherence in the treatment of bipolar disorder.

\section{Objectives}

- To describe the modifiable determinants of medication adherence from the perspectives of patients, carers, healthcare professionals and any other third parties.

- To map reported modifiable determinants of medication adherence to the domains of the TDF.

\section{METHOD}

This research protocol is based on ENhancing Transparency in REporting the synthesis of Qualitative research (ENTREQ $)^{28}$ and Preferred Reporting Items for Systematic Reviews and Meta-Analyses Protocols (PRISMA-P). ${ }^{29}$ The protocol is registered with PROSPERO- www.crd. york.ac.uk/PROSPERO/—international prospective register of systematic reviews.

\section{Evidence synthesis}

We will use the TDF as an a priori framework for our review. We will map the extracted modifiable determinants of adherence from the included studies to the domains of the TDF. The deductive approach of this framework synthesis method ${ }^{30-33}$ has the potential to restrict the nature of identified determinants. However, the comprehensive nature of the TDF should enable identification of all determinants relevant to behaviour change and any determinants that cannot be mapped to a TDF domain will still be extracted and mapped to new domains if appropriate. ${ }^{34} \mathrm{~A}$ further benefit of mapping determinants to the TDF is its linkage to behaviour change techniques. ${ }^{17}$ This approach was successfully applied by Allemann and colleagues to match adherence interventions to determinants of patient adherence. ${ }^{27}$ This early identification of relevant behaviour change techniques affords a substantial advantage in terms of informing the design of theory and evidencebased medication adherence interventions for people under prescribed medication for bipolar disorder.

\section{Approach to searching, search strategy and data sources}

We will employ a preplanned search strategy to seek all relevant studies. Our search strategy will consist of three parameters: disease (bipolar disorder), treatment (medication) and outcome (adherence). Following a scoping exercise of search terms (on Pubmed, Medline and Embase) to define our search strategy, we decided to use the MeSH (Medical Subject Heading) terms 'Treatment Adherence and Compliance', 'Bipolar Disorder' and 'Psychotropic Drugs' for our search. We will adapt these search terms for the databases that do not permit MeSH terms or use different MeSH terms.

We will search the following databases: CINAHL, Cochrane Library (CENTRAL), Embase, LiLACS, Medline, PsychINFO, Pubmed and the reference list of all included studies will be reviewed for further relevant studies.

\section{Study inclusion criteria}

We will include any primary studies; both qualitative and quantitative for example, focus groups, interviews and surveys; explicitly reporting one or more modifiable determinants of medication adherence in the maintenance treatment of bipolar disorders from the perspective of patients, carers, healthcare professionals or any other third parties. There will be no date restrictions but we will include only the studies published in English language. We will include studies of patients aged 18 years and above with bipolar disorder with or without other comorbidities including dual diagnosis, other mental or physical health conditions to represent the real-world patient population. We will exclude reviews, intervention studies to improve adherence, case reports, letters, editorials, commentaries, opinion pieces, clinical guidelines or general disease management articles and studies not involving humans. We will also exclude studies involving short-term treatment of acute agitation or treatment other than medication such as psychotherapy. Studies where effect of individual barriers/facilitators to adherence could not be isolated/extracted from composite measures (such as adherence rating scale) will be excluded.

\section{Study screening methods}

We will use computer software Covidence, ${ }^{35}$ an online systematic review program, for screening retrieved studies. Screening of studies for inclusion in this review will involve three distinct stages:

I. Title Screening: After removal of duplicates using the reference manager software Mendeley, the remaining studies will be screened for their relevance to the review. Definite non-relevant studies will be excluded while relevant, or unclear studies, will be retained for abstract screening.

II. Abstract Screening: Abstracts of the remaining studies will be screened by the primary reviewer (AP) and second reviewers (CG, DB, FS, GM, JW and SS) independently to identify studies that potentially meet the inclusion criteria outlined earlier. Any 
disagreement between the two reviewers will be resolved through further discussion and referral to a third reviewer (DB) if there is a failure to achieve agreement.

III. Full Article Screening: Full articles will be reviewed independently by two reviewers (AP, CG, DB, FS, GM, JW and SS) using predefined inclusion/exclusion criteria. Any disagreement between two reviewers will be resolved through discussion or the involvement of the third reviewer. We will use Cohen's kappa to report the level of agreement between first and second reviewers.

Within published syntheses of qualitative research, there is often a lack of transparency about the search processes employed, with neither the search strategy nor databases detailed. ${ }^{28}$ For a comprehensive approach, we will use the PRISMA flowchart for reporting different phases of searching, screening and identifying studies for inclusion in the qualitative synthesis as recommended by ENTREQ ${ }^{28}$

\section{Data extraction and mapping}

We will use the computer software program Nvivo V.12 $2^{36}$ to extract data and to map the modifiable determinants of medication adherence to the domains of the TDF. While medication adherence is generally described as taking $\geq 80 \%$ doses of prescribed medications, some studies report adherence in gradient terms (eg, good, moderate, low adherence and non-adherence). ${ }^{4}$ Yet, in some cases (eg, in HIV), adherence means taking $\geq 95 \%$ doses of prescribed medications. ${ }^{36}$ Acknowledging this wide variation on definition of medication adherence, we will report the definition used for adherence in included studies for transparency and comparison among studies. Extracted information will include study characteristics (eg, title, year of publication, country, study design, population, number of participants, definition and rate of adherence) and modifiable determinants of medication adherence in patients with bipolar disorder.

We will map each extracted determinant to one of the following domains of the TDF: (1) Knowledge, (2) Skills, (3) Social Influences, (4) Memory, Attention and Decision Processes, (5) Behavioural Regulation, (6) Professional/Social Role and Identity, (7) Beliefs about Capabilities, (8) Belief about Consequences, (9) Optimism, (10) Intentions, (11) Goals, (12) Emotion, (13) Environmental Context and Resources and (14) Reinforcement. We will use constructs within the domains and construct definitions of the $\mathrm{TDF}^{21}$ to inform mapping decisions. Any determinants that do not fit within the existing domains will be organised into 'Others' domain. ${ }^{34}$

Within Nvivo V.12, we will create four themes in line with the aim of this study:

1. Patient Perspective.

2. Carers Perspective.

3. HealthCare Professional Perspective.
4. Others Perspectives.

Within each theme we will create two subthemes (Barriers and Facilitators), and within each of these subthemes we will create 15 domains (14 TDF domains plus 'Others').

Two reviewers will pilot data extraction and coding of determinants of adherence to the domains of TDF from four studies. For example, if the following text was extracted from a study 'Forgetting to take medication or being careless at times about taking medication was reported to be experienced by x participants', this would be coded to the TDF domain 'Memory, attention and decision process'. The reviewers will then compare and discuss their coding to generate consensus in interpretation of literature identified determinants. After piloting, all data will be extracted by one reviewer and independently checked by second reviewer for completeness.

All extracted determinants will be independently mapped onto the 14 domains of the TDF or 'Others' category by two reviewers. The two reviewers will meet and discuss their mapping regularly. Any disagreement in mapping will be resolved through discussion between the two reviewers and referral to a third reviewer as adjudicator if the two reviewers fail to agree. We will use Cohen's kappa to report agreement between the first and second reviewers as we are dealing with nominal data, that is, agreement and not with the domain to which a determinant is mapped onto the TDF.

\section{Quality assessment}

No studies will be excluded based on quality as our aim is to identify determinants of medication adherence as comprehensively as possible. However, we will undertake a quality assessment for the purposes of characterising included studies. There is neither gold-standard tool for any study design nor is there any widely accepted generic quality assessment tool that functions across multiple study types. ${ }^{38}$ We will use bespoke Critical Appraisal Skills Programme qualitative (CASP), ${ }^{39}$ Critical appraisal of survey ${ }^{40}$ and Cochrane risk of bias tool $^{41}$ to critically appraise qualitative studies, surveys and trials, respectively. These tools meet the requirements of the study and provide key quality criteria such as validity, reliability and objectivity. ${ }^{42}$ Quality assessment will be carried out by two independent reviewers. Any disagreement between reviewers will be resolved through discussion and, if necessary, referral to a third reviewer for arbitration.

\section{Patient and public involvement}

This systematic review is a part of the C-MAB project that include three patients and a carer as research advisory board members. Patient and public involvement (PPI) has influenced the study design with two notable recommendations: inclusion of the carer's perspective on medication adherence and differentiating between modifiable and non-modifiable determinants of medication 
adherence. Two PPI representatives (CG and RG) are listed as authors.

\section{ETHICS AND DISSEMINATION}

Ethical approval is not required as primary data will not be collected. The results will be disseminated through a peer-reviewed publication.

\section{Author affiliations}

${ }^{1}$ Pharmacy, Norfolk and Suffolk NHS Foundation Trust, Norwich, UK

${ }^{2}$ School of Pharmacy, University of East Anglia, Norwich, UK

${ }^{3}$ Health Services and Performance Research EA 7425 HESPER, University Claude

Bernard Lyon 1, Lyon, France

${ }^{4}$ Norwich Medical School, Norwich, UK

${ }^{5}$ Patient and Carer Representatives, Norwich, UK

${ }^{6}$ Faculty of Medicine, Harvard Medical School, Boston, Massachusetts, USA

${ }^{7}$ Norfolk and Suffolk NHS Foundation Trust, Norwich, UK

Correction notice This article has been corrected since it was published. Middle name and affiliation has been updated for Dr Alexandra Lelia Dima. Funding information has also been updated.

Contributors All authors (ARP, AD, ABC, CGa, CGi, RG, GM, SS, FS, BT, MJT, JW and $\mathrm{DB}$ ) helped conceive the study, reviewed the protocol and provided intellectual critique. ARP and DB designed and wrote the protocol. ARP registered the study with PROSPERO. All authors (ARP, AD, ABC, CGa, CGi, RG, GM, SS, FS, BT, MJT, JW and $\mathrm{DB}$ ) have approved the publication of this protocol.

Funding This research is a part of the Clinical Doctoral Research Fellowship program funded by Health Education England / National Institute of Health Research. AD has been funded by the European Commission (MSCA-IF n ${ }^{\circ} 706028$ ) during the preparation of this manuscript.

Competing interests None declared.

Patient consent for publication Not required.

Provenance and peer review Not commissioned; externally peer reviewed.

Open access This is an open access article distributed in accordance with the Creative Commons Attribution Non Commercial (CC BY-NC 4.0) license, which permits others to distribute, remix, adapt, build upon this work non-commercially, and license their derivative works on different terms, provided the original work is properly cited, appropriate credit is given, any changes made indicated, and the use is non-commercial. See: http://creativecommons.org/licenses/by-nc/4.0/.

\section{REFERENCES}

1. National Institute for Health and Care Excellence [NICE]. Bipolar Disorder: Assessment and Management [Internet]. NICE clinical guideline. 2014. https://www.nice.org.uk/guidance/cg185

2. Gitlin M, Frye MA. Maintenance therapies in bipolar disorders. Bipolar Disord 2012;14(Suppl 2):51-65.

3. Yatham LN, Kennedy SH, Parikh S V, et al. Long-acting injectable antipsychotics for the maintenance treatment of bipolar disorder. JÖRESKOG KG, editor. J Clin Psychiatry 2014;9:392-400.

4. De Geest S, Sabaté E. Adherence to long-term therapies: evidence for action. Eur J Cardiovasc Nurs 2003;2:323.

5. Samalin L, Nourry A, Charpeaud T, et al. What is the evidence for the use of second-generation antipsychotic long-acting injectables as maintenance treatment in bipolar disorder? Nord J Psychiatry 2014;68:227-35.

6. Gigante AD, Lafer B, Yatham LN. Long-acting injectable antipsychotics for the maintenance treatment of bipolar disorder. CNS Drugs 2012;26:403-20.

7. Scott J, Pope M. Self-reported adherence to treatment with mood stabilizers, plasma levels, and psychiatric hospitalization. $A m \mathrm{~J}$ Psychiatry 2002;159:1927-9.

8. Easthall C, Taylor N, Bhattacharya D. Barriers to medication adherence in patients prescribed medicines for the prevention of cardiovascular disease: a conceptual framework. Int J Pharm Pract 2018.
9. Vrijens B. Medication Adherence: State of the Art [Internet]. 2016. http://acto.unamur.be/1st-acto-conference/documents-downloads/ vrijens-presentation (cited 22 Aug 2018).

10. Oxford English Dictionary Online: Oxford University Press, 2017.

11. Craig P, Dieppe P, Macintyre S, et al. Developing and evaluating complex interventions: the new Medical Research Council guidance. BMJ 2008;337:a1655.

12. National Institute for Health and Care Excellence. Behaviour change: general approaches (PH6) [Internet]. 2007. https://www.nice.org.uk/ Guidance/PH6

13. Nguyen TM, La Caze A, Cottrell N. What are validated self-report adherence scales really measuring?: a systematic review. Br J Clin Pharmacol 2014;77:427-45.

14. García S, Martínez-Cengotitabengoa M, López-Zurbano S, et al. Adherence to antipsychotic medication in bipolar disorder and schizophrenic patients: a systematic review. J Clin Psychopharmacol 2016;36:355-71.

15. Goodwin GM, Haddad PM, Ferrier IN, et al. Evidencebased guidelines for treating bipolar disorder: Revised third edition recommendations from the British Association for Psychopharmacology. J Psychopharmacol 2016;30:495-553.

16. Grunze H, Vieta E, Goodwin GM, et al. The World Federation of Societies of Biological Psychiatry (WFSBP) guidelines for the biological treatment of bipolar disorders: update 2012 on the long-term treatment of bipolar disorder. World J Biol Psychiatry 2013;14:154-219.

17. Ralat SI, Depp CA, Bernal G. Reasons for nonadherence to psychiatric medication and cardiovascular risk factors treatment among latino bipolar disorder patients living in puerto rico: a qualitative study. Community Ment Health J 2018:54:707-16.

18. Velligan DI, Weiden PJ, Sajatovic M, et al. The expert consensus guideline series: adherence problems in patients with serious and persistent mental illness. J Clin Psychiatry 2009;70(Suppl 4):1-8.

19. Kane JM, Kishimoto T, Correll CU. Non-adherence to medication in patients with psychotic disorders: epidemiology, contributing factors and management strategies. World Psychiatry 2013;12:216-26.

20. Sendt KV, Tracy DK, Bhattacharyya S. A systematic review of factors influencing adherence to antipsychotic medication in schizophreniaspectrum disorders. Psychiatry Res 2015;225:14-30.

21. Cane J, O'Connor D, Michie S. Validation of the theoretical domains framework for use in behaviour change and implementation research. Implement Sci 2012;7:37.

22. Michie S, Johnston M, Francis J, et al. From theory to intervention: mapping theoretically derived behavioural determinants to behaviour change techniques. Appl Psychol 2008:57:660-80.

23. Devine F, Edwards T, Feldman SR. Barriers to treatment: describing them from a different perspective. Patient Prefer Adherence 2018;12:129-33.

24. Baldessarini RJ, Perry R, Pike J. Factors associated with treatment nonadherence among US bipolar disorder patients. Hum Psychopharmacol 2008;23:95-105.

25. Byerly M, Fisher R, Whatley K, et al. A comparison of electronic monitoring vs. clinician rating of antipsychotic adherence in outpatients with schizophrenia. Psychiatry Res 2005;133:129-33.

26. Tacchi MJ SJ. Improving Adherence in Schizophrenia and Bipolar. Chichester, England: John Wiley \& Sons, 2005.

27. Allemann SS, Nieuwlaat R, van den Bemt BJ, et al. Matching Adherence Interventions to Patient Determinants Using the Theoretical Domains Framework. Front Pharmacol 2016;7.

28. Tong A, Flemming $\mathrm{K}$, Mclnnes $\mathrm{E}$, et al. Enhancing transparency in reporting the synthesis of qualitative research: ENTREQ. BMC Med Res Methodol 2012;12:181.

29. Shamseer L, Moher D, Clarke M, et al. Preferred reporting items for systematic review and meta-analysis protocols (PRISMA-P) 2015: elaboration and explanation. BMJ 2015;349:g7647.

30. Dixon-Woods M. Using framework-based synthesis for conducting reviews of qualitative studies. BMC Med 2011:9:39.

31. Barnett-Page E, Thomas J. Methods for the synthesis of qualitative research: a critical review. BMC Med Res Methodol 2009;9:59.

32. Pope C, Ziebland S, Mays N, et al. Qualitative research in health care. Analysing qualitative data. BMJ 2000;320:114-6.

33. Carroll C, Booth A, Cooper K. A worked example of "best fit" framework synthesis: a systematic review of views concerning the taking of some potential chemopreventive agents. BMC Med Res Methodol 2011;11:29.

34. Taylor N, Lawton R, Conner M. Development and initial validation of the determinants of physical activity questionnaire. Int $J$ Behav Nutr Phys Act 2013;10:74

35. Covidence Systematic Review Software. Melbourne, Australia: Veritas Health Innovation, 2018. Available from. www.covidence.org 
36. Nvivo. Nvivo 12 for Windows, NVivo qualitative data analysis Software: QSR International Pty Ltd, 2018.

37. Achappa B, Madi D, Bhaskaran U, et al. Adherence to antiretroviral therapy among people living with HIV. N Am J Med Sci 2013;5:220-3.

38. Katrak P, Bialocerkowski AE, Massy-Westropp N, et al. A systematic review of the content of critical appraisal tools. BMC Med Res Methodol 2004;4:22.

39. Critical Appraisal Skills Programme (CASP). 10 Questions To Help You Make Sense of Qualitative Research. https://casp-uk.net/wpcontent/uploads/2018/03/CASP-Qualitative-Checklist-Download.pdf

40. Center for, Evidence Based Management. Adapted from Crombie, The Pocket Guide to Critical Appraisal; the critical appraisal approach used by the Oxford Centre for Evidence Medicine, checklists of the Dutch Cochrane Centre, BMJ editor's checklists and the checklists of the EPPI Centre. http://www.cebma.org/wp-content/uploads/CriticalAppraisal-Questions-for-a-Survey.pdf (cited 1 Aug 2018)

41. Higgins JP, Altman DG, Gøtzsche PC, et al. The Cochrane Collaboration's tool for assessing risk of bias in randomised trials. BMJ 2011;343:d5928.

42. Frambach JM, van der Vleuten CP, Durning SJ. AM last page. Quality criteria in qualitative and quantitative research. Acad Med 2013;88:552. 